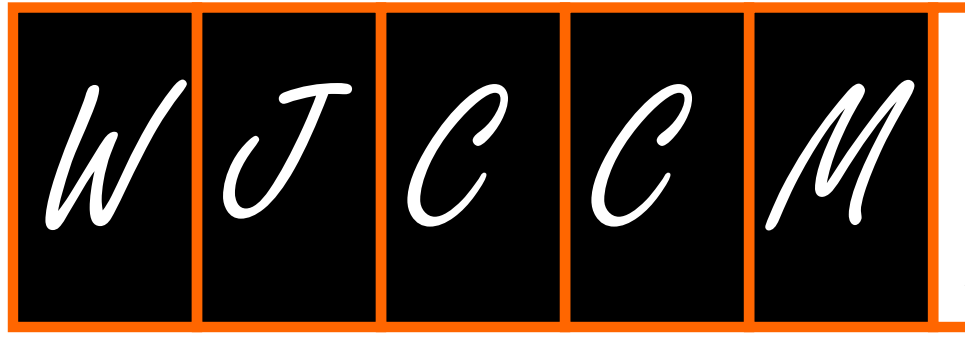

Submit a Manuscript: https:/ / www.f6publishing.com

\section{World Journal of Critical Care Medicine}

World J Crit Care Medl 2019 September 11; 8(5): 72-81

\title{
Tuberculosis septic shock, an elusive pathophysiology and hurdles in management: A case report and review of literature
}

\author{
Rashmi Mishra, Harish K Patel, Rakesh Singasani, Trupti Vakde
}

ORCID number: Rashmi Mishra (0000-0001-6747-6490); Harish Patel (0000-0003-3638-9495); Rakesh Singasani (0000-0001-5094-7663); Trupti Vakde (0000-0002-5154-3051).

\begin{abstract}
Author contributions: All authors have reviewed the literature and contributed to manuscript drafting. Vakde T, Mishra R and Patel HK were responsible for the revision of the manuscript for important intellectual content; All authors issued final approval for the version to be submitted.

Informed consent statement: All patients have deceased, as per the recommendation of the editorial office, the initial consent for the treatment and hospitalization have been uploaded.
\end{abstract}

Conflict-of-interest statement: The authors declare that they have no conflict of interest.

CARE Checklist (2016) statement: The authors have read the CARE Checklist (2013), and the manuscript was prepared and revised according to the CARE Checklist (2016).

Open-Access:This article is an open-access article which was selected by an in-house editor and fully peer-reviewed by external reviewers. It is distributed in accordance with the Creative Commons Attribution Non Commercial (CC BY-NC 4.0) license, which permits others to distribute, remix, adapt, build upon this work non-commercially, and license their derivative works on different terms, provided the
Rashmi Mishra, Pulmonary and Critical Care, Penn Highlands Healthcare, Dubois, PA 15801, United States

Harish K Patel, Division of Gastroenterology, Department of Medicine, Bronx Care Health system, Bronx, NY 10457, United States

Rakesh Singasani, Department of Medicine, SBH Health System, Bronx, NY 10457, United States

Trupti Vakde, Division of Pulmonary and Critical Care, Department of Medicine, Bronx Care Health System, Bronx, NY 10457, United States

Corresponding author: Trupti Vakde, MD, Attending Doctor, Attending Physician, Division of Pulmonary and Critical Care, Department of Medicine, Bronx Care Health System, 1650 Grand concourse, Bronx, NY 10457, United States. tvakde@bronxcare.org

Telephone: +1-718-9601234

Fax: $+1-917-7780876$

\section{Abstract}

\section{BACKGROUND}

Tuberculosis (TB) is a rare etiology of the septic shock. Timely administration of the anti-microbial agents has shown mortality benefit. Prompt diagnosis and a high index of suspicion are crucial to the management. We present three cases of TBSS with poor outcome in the majority despite timely and susceptible antibiotic administration.

\section{CASE SUMMARY}

Sixty-seven-year-old woman with latent TB presented with fever, cough, and shortness of breath. She was promptly diagnosed with active TB and started on the appropriate anti-microbial regimen; she had a worsening clinical course with septic shock and multi-organ failure after initiation of antibiotics. Thirty-threeyear-old man immunocompromised with acquired immune deficiency syndrome presented with fever, anorexia and weight loss. He had no respiratory symptoms, and first chest X-ray was normal. He had enlarged liver, spleen and lymph nodes suspicious for lymphoma. Despite broad-spectrum antibiotics, he succumbed to refractory septic shock and multi-organ failure. It was shortly before his death that anti-TB antimicrobials were initiated based on pathology reports of bone marrow and lymph node biopsies. Forty-nine-year-old woman with asthma and latent TB admitted with cough and shortness of breath. Although Initial sputum analysis was negative, a subsequent broncho-alveolar lavage turned out to be 
original work is properly cited and the use is non-commercial. See: http://creativecommons.org/licen ses/by-nc/4.0/

Manuscript source: Unsolicited manuscript

Received: April 6, 2019

Peer-review started: April 8, 2019

First decision: August 2, 2019

Revised: August 13, 2019

Accepted: August 21, 2019

Article in press: August 21, 2019

Published online: September 11, 2019

P-Reviewer: Mousa HAL

S-Editor: Yan JP

L-Editor: A

E-Editor: Liu MY positive for acid fast bacilli followed by initiation of susceptible ant-TB regimen. She had a downward spiral clinical course with shock, multi-organ failure and finally death.

\section{CONCLUSION}

Worse outcome despite timely initiation of appropriate antibiotics raises suspicion of TB immune reconstitution as a possible pathogenesis for TB septic shock.

Key words: Tuberculosis septic shock; Tuberculosis and immune reconstitution; Tuberculosis in intensive care unit; Case fatality for tuberculosis septic shock; Case report

CThe Author(s) 2019. Published by Baishideng Publishing Group Inc. All rights reserved.

Core tip: Tuberculosis septic shock is a rare entity. We present three cases of tuberculosis septic shock with varied clinical manifestations. Mycobacterium tuberculosis culture or nucleic acid amplification testing confirmed diagnosis of tuberculosis. All of our presented cases had poor outcome despite timely administration of appropriate antituberculosis regimen. There was clinical and radiological deterioration after administration of anti-microbial agents. This deteriorating clinical course raises a concern for immune reconstitution as possible pathogenesis for tuberculosis septic shock.

Citation: Mishra R, Patel HK, Singasani R, Vakde T. Tuberculosis septic shock, an elusive pathophysiology and hurdles in management: A case report and review of literature. World $J$ Crit Care Med 2019; 8(5): 72-81

URL: https://www.wjgnet.com/2220-3141/full/v8/i5/72.htm

DOI: https://dx.doi.org/10.5492/wjccm.v8.i5.72

\section{INTRODUCTION}

Sepsis is a significant burden on healthcare across the globe ${ }^{[1]}$. In United States, there are 970000 cases of sepsis annually[2]. In United States overall sepsis, related mortality is $12.5 \%$. The severity of sepsis affects outcomes, with $35 \%$ mortality in septic shock ${ }^{[2]}$. Prompt diagnosis and early treatment is key to management. Any delay in antibiotics administration is associated with the worsening of sepsis severity ${ }^{[3]}$.

Martin et al ${ }^{[4]}$ analyzed 2.6 million sepsis cases from the premier database in United States, for six years. Fifteen percent of patients did not manifest sepsis at the time of hospitalization. The mortality outcomes were worst in this group of patients. The group of patients with sepsis at presentation have lower mortality. This improved outcome may be attributed to the timely diagnosis and prompt antibiotics administration.

Empiric antibiotic administration within one hour of presentation has been proven to lower sepsis-related mortality ${ }^{[5]}$. Broad spectrum anti-microbial agents are selected based on the most common gram positive and gram negative bacterial infection ${ }^{[6]}$. Respiratory tract infection is the most common etiology for sepsis and septic shock ${ }^{[7]}$. Tuberculosis (TB) is an uncommon but well-recognized etiology of sepsis and is seldom discussed in the western population.

Mycobacterium tuberculosis is an acid-fast bacteria with a predominant pulmonary presentation $^{[8]}$, though not a frequent cause of pneumonia in the western population. Only twenty percent of the cases have a sole extra-pulmonary manifestation ${ }^{[8]}$. The incidence of TB septic shock (TBSS) though not reported, is sporadic. The Center for Disease Control and Prevention have reported a declining incidence in the diagnosis of $\mathrm{TB}^{[9]}$. The rarity of TBSS makes this case series novel. We describe three cases of TBSS that presented in our institution. Despite the diagnosis of sepsis at presentation, case fatality rate is very high, as opposed to the general expectation in sepsis epidemiology ${ }^{[2]}$. Each case is unique with its presentation, and this case series provides an excellent opportunity to analyze the demographic features, clinical characteristics, radiologic and laboratory findings as well as the pitfalls in the management of patients with TBSS. 


\section{CASE PRESENTATION}

\section{Case 1}

Chief complaint: A 67-year-old female presented to the emergency department with progressively worsening shortness of breath and fever.

History of present illness: The patient complained of twenty pounds' weight loss over three months. She had shortness of breath, fever and productive cough for a duration of four weeks.

History of past illness: She had medical conditions of hypertension, chronic obstructive pulmonary disease, gastroesophageal reflux disease, gout and a 40-pack year history of smoking. She lived at home and had a history of exposure to TB when she was 9 years old as well as a known positive tuberculin skin test. She had never traveled outside the country.

Physical examination: Her vital signs were significant for low-grade fever of $37.9^{\circ} \mathrm{C}$, heart rate of 110 beats per minute, blood pressure of 165/100 $\mathrm{mm}$ of $\mathrm{Hg}$ and oxygen saturation of $96 \%$ on room air. She had bilateral rales on lung auscultation and rest of the physical exam was unremarkable

Laboratory examinations: Blood analysis was significant for hyponatremia (sodium $118 \mathrm{mEq} / \mathrm{L}$ ) and anemia (Hemoglobin $7.3 \mathrm{~g} / \mathrm{dL}$ ).

Imaging examinations: Chest $X$-ray and computed tomography (CT) scan on admission showed bilateral patchy infiltrates predominantly in the upper lobes (Figure $1 \mathrm{~A}$ and $\mathrm{B}$ ).

Treatment: Ceftriaxone and azithromycin were initiated for community-acquired pneumonia (CAP), and she was admitted to intensive care unit (ICU) for sepsis and hyponatremia. She was also placed in respiratory isolation due to high suspicion for TB. On day 2 of admission, sputum acid fast bacilli (AFB) stain was reported positive, and the patient was started on isoniazid, rifampin, ethambutol, and pyrazinamide (RIPE) for Mycobacterium tuberculosis. Human immunodeficiency virus (HIV) test was negative. Mycobacterium tuberculosis was confirmed by polymerase chain reaction (PCR) before the final culture report. Subsequently, she became progressively more hypoxic and hypotensive requiring mechanical ventilation and vasopressor support. Chest X-ray after intubation showed worsening of bilateral lung infiltrates (Figure 1C). She developed multi-organ failure secondary to shock including hepatic and renal dysfunction requiring hemodialysis. Patient was also initiated on high dose steroids, and antibiotic coverage was broadened with no significant improvement in hemodynamic status.

Final diagnosis: Septic shock due to Mycobacterium tuberculosis.

Outcome and follow up: Due to poor prognosis and no improvement in the patient's condition, the family wished for transfer to hospice care and patient died on day 22 of hospitalization. Final culture and susceptibility reports confirmed sensitivity to RIPE.

Case 2

Chief complaints: A 33-year-old man was called to come to the emergency department for abnormal laboratory results.

History of present illness: In the emergency department he reported subjective fever, poor appetite and weight loss for one month. He also reported diarrhea for past few days but no abdominal pain, nausea, vomiting, cough or shortness of breath.

History of past illness: Patient had known history of HIV/acquired immune deficiency syndrome (AIDS) not adherent to antiretroviral medications with very high viral load and CD4 count of less than 20, past intravenous drug use and active tobacco dependence. He had history of anemia and deep vein thrombosis and was living in a nursing home for few months prior to presentation. He was born in Puerto Rico and came to the Unites States a year ago.

Physical examination upon admission: His vital signs were significant for hypotension with blood pressure of $91 / 60 \mathrm{mmHg}$, tachycardia with heart rate of 130 beats per minute and fever with temperature of $39.4^{\circ} \mathrm{C}$. On physical examination he was lethargic and confused. Hypotension initially improved with intravenous fluids

Laboratory examinations: Initial laboratory data was significant for leukocytosis of 31151/ $\mu \mathrm{L}$ and hyponatremia (sodium $121 \mathrm{mEq} / \mathrm{L}$ ). 

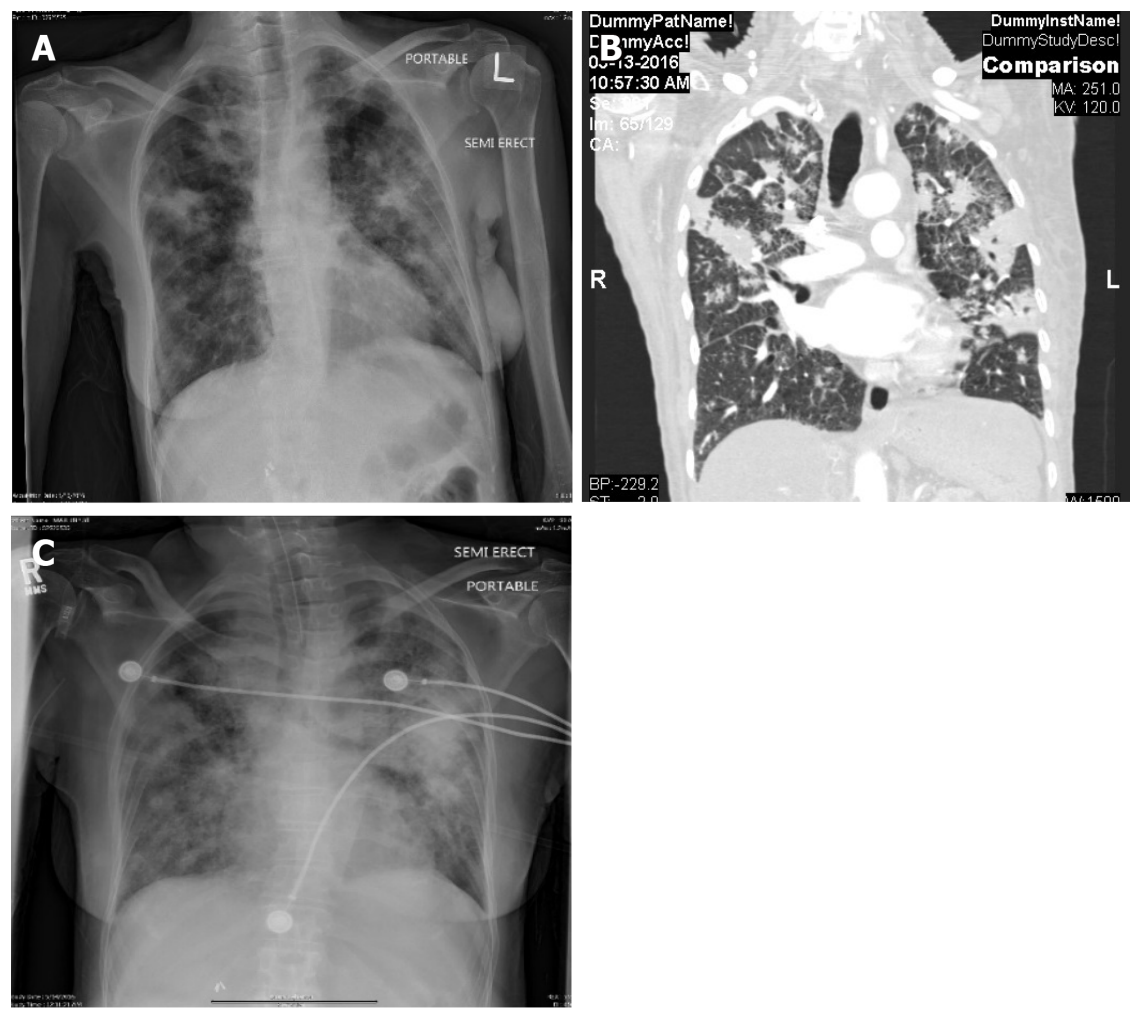

Figure 1 Chest X-ray and computed tomography (case 1). A: Chest X-ray demonstrating the bilateral patchy infiltrates at the time of admission; $B$ : Computed tomography scans of chest with coronal section demonstrating bilateral patchy infiltrates at the time of admission; $\mathrm{C}$ : X-ray chest demonstrating worsening of the bilateral infiltrates after intubation and initiation of anti tuberculosis medications.

Imaging examinations: Chest X-ray on admission showed no infiltrates (Figure 2A). Ultrasound of abdomen was significant for hepatosplenomegaly, ascites and enlarged peri-pancreatic lymph nodes

Treatment: Patient was admitted to the critical care unit with a presumptive diagnosis of sepsis and initiated on the broad spectrum antibiotics (vancomycin and piperacillin-tazobactam). He was started on metronidazole for suspected Clostridium difficile colitis. Subsequently he developed acute hypoxic respiratory failure and septic shock requiring intubation and vasopressor support. Chest X-ray after intubation showed new bilateral infiltrates (Figure 2B). Blood cultures were negative. He underwent lumbar puncture which showed an opening pressure of $35 \mathrm{~cm} \mathrm{of} \mathrm{H}_{2} \mathrm{O}$, red blood cell (RBC) of 1825 cells/dL, white blood cell count of 1 cell/dL, glucose of 65 $\mathrm{mg} / \mathrm{dL}$ and protein $36 \mathrm{mg} / \mathrm{dL}$. In view of significant RBC count in cerebrospinal fluid (CSF) and high opening pressure, he was also initiated on acyclovir as empiric treatment for herpes simplex encephalitis. Broncho alveolar lavage (BAL) was negative for bacterial, viral, fungal and Pneumocystis jirovecii cultures. Initial smears for AFB were negative. The CSF bacterial cultures and herpes simplex virus PCR were negative. He was initiated on antiretroviral therapy on day 8 of hospitalization after preliminary cultures were negative. In view of persistent fever spikes and lymphadenopathy, hematology consultation was obtained and patient underwent bone marrow biopsy, and right axillary lymph node biopsy. Caspofungin was added to his antibiotic regimen for refractory septic shock and persistent fever. Bone marrow biopsy was positive for acid fast organisms. Pathology of right axillary lymph node biopsy reported as necrotizing granulomas with mycobacteria. An empiric treatment for Mycobacterium tuberculosis and mycobacterium avium complex with Isoniazide, Ethambutol, Rifabutin, pyrazinamide and clarithromycin was initiated awaiting final confirmation

Final diagnosis: Septic shock due to Mycobacterium tuberculosis.

Outcome and follow up: Patient died secondary to worsening shock and multi organ failure after $22 \mathrm{~d}$ of admission. Sputum, BAL and peritoneal fluid cultures were reported positive for Mycobacterium tuberculosis complex post mortem and susceptibility reports confirmed no resistance. 

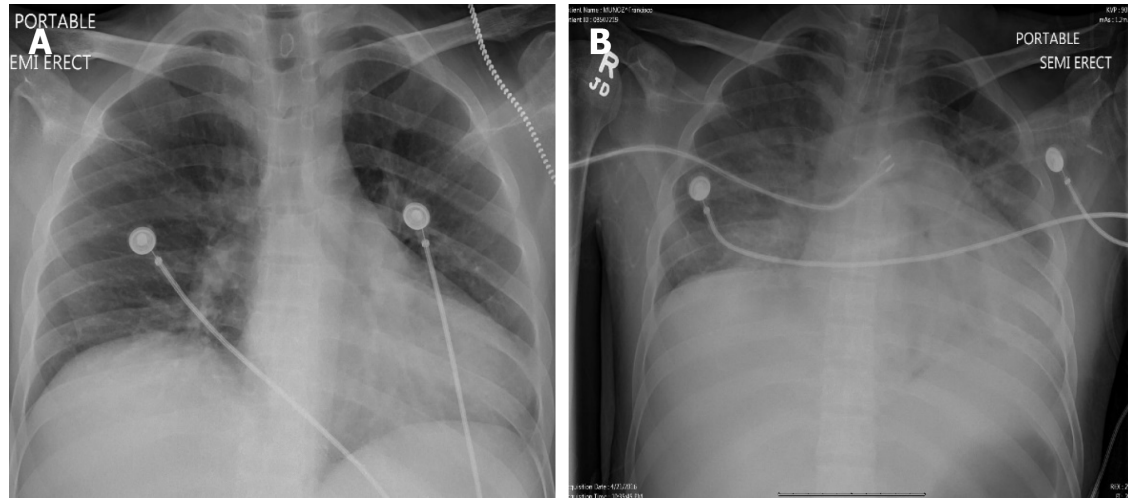

Figure 2 X-ray chest (case 2). A: X-ray chest with no infiltrates at the time of admission; B: X-ray chest with new infiltrates and hypoxia requiring intubation and mechanical ventilation.

\section{Case 3}

Chief complaints: A 49-year-old woman presented to the hospital with productive cough for three to four weeks.

History of present illness: Patient presented with worsening cough, shortness of breath and wheezing. She denied fever, chills, weight loss, recent travel or sick contacts.

History of past illness: Her medical conditions included hypertension, latent TB (treated for 6 mo in 2007 as per patient), Asthma and smoking.

Physical examination upon admission: Vital signs were significant for mild tachycardia with heart rate of 114 beats per minute. She was afebrile with normal blood pressure and oxygen saturation of $98 \%$ on room air. There was bilateral wheezing on auscultation and rest of the examination was unremarkable.

Laboratory examinations: Initial blood analysis revealed leukocytosis with white blood cell count of 11300 cells / $\mu$ L. Her electrolytes and liver function tests were normal except a high alkaline phosphatase level of 111 unit/dL.

Imaging examinations: Chest $X$-ray on admission showed a stable left upper lobe thick walled cavity and new left lower lobe infiltrates in comparison to the chest X-ray done few weeks ago (Figure 3A). CT of the chest was done which revealed additional left lung thick walled cavities and multiple nodules on both sides (Figure 3B).

Treatment: Given the high suspicion for TB she was placed on respiratory isolation, and started on broad spectrum antibiotics with intravenous piperacillin-tazobactam and vancomycin. Three sputum samples were initially negative for AFB. Differential diagnoses included pulmonary $\mathrm{TB}$, atypical mycobacteria and fungal infections. She underwent bronchoscopy, BAL and trans bronchial biopsy. The BAL stains were positive for AFB and mycobacterium TB was identified with DNA probe and subsequent cultures. Cultures from BAL also grew pseudomonas aeruginosa in low colony counts of 1000-9000 CFU/mL. Pseudomonas was pan sensitive. Antibiotics were titrated, with initiation of RIPE and discontinuation of vancomycin.

Final diagnosis: Septic shock due to Mycobacterium tuberculosis.

Outcome and follow up: On day 6 of admission, her respiratory status declined to require intubation for hypoxic respiratory failure and transfer to ICU. She developed worsening bilateral infiltrates (Figure 3C), hyponatremia (sodium $125 \mathrm{mEq} / \mathrm{L}$ ) and septic shock. She remained in shock and died on day 12 of hospitalization.

\section{DISCUSSION}

There is ample literature to support the case fatality of TBSS ${ }^{[10]}$. It is evident that prompt identification and early antibiotic administration improves mortality. The mortality risk factors of TB patient who are critically ill have been identified ${ }^{[11]}$. However, given the scantiness of TBSS, no standard protocol is constructed to improve the outcomes of this rare entity. We reviewed our cases and analyzed them to determine the hurdles in the management of TBSS. To better understand the 

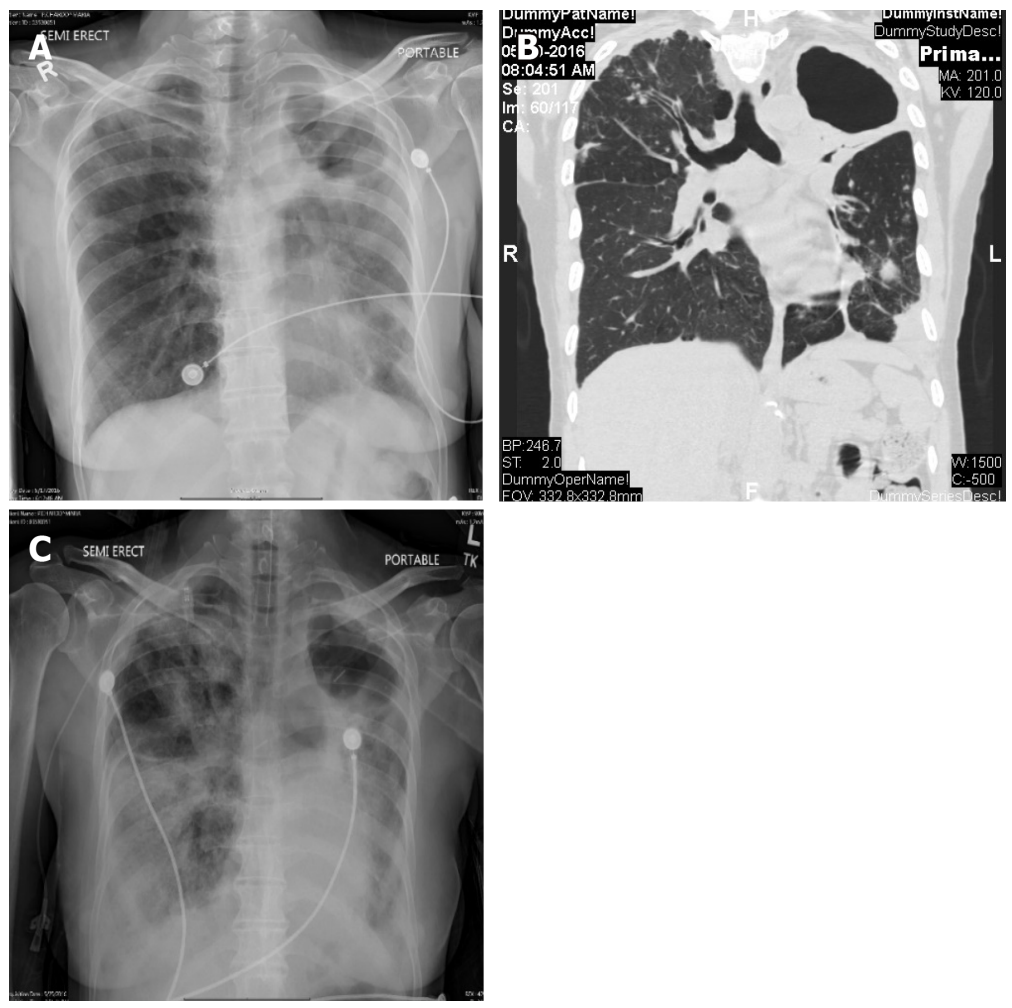

Figure 3 X-ray chest and computerized axial tomography (case 3). A: X-ray chest revealing the left upper lobe cavity and left lower lobe infiltrates at time of admission; $B$ : Computerized axial tomography scan of the chest at time of admission revealing left lung thick walled cavities and multiple nodules on both side; $\mathrm{C}$ : $\mathrm{X}$-ray chest revealing worsening of the bilateral infiltrate after intubation and of the anti-tuberculosis medications.

management of TBSS we divided it into several categories. This includes understanding pathophysiology of TBSS, high risk clinical features, modalities for prompt diagnosis and utility of empiric TB treatment based on clinical suspicion.

TBSS is extremely rare. Kethireddy et al ${ }^{[10]}$, in a limited institutional review across the globe, could identify TBSS as etiology of septic shock in 1\% of the cases in a pool of 5419 patients. A Majority of them were identified outside the United States. The incidence in United States was almost undetectable. Given the clinical rarity of TB in a low prevalence area ${ }^{[10]}$ the suspicion for diagnosis is low. Hence areas of high prevalence of TB should be identified. California leads the TB incidence rate in United States, followed by Texas, New York, and Florida ${ }^{[12]}$. Over a one-year duration in New York, there was a 10\% increase in the number of TB cases from 2016 to $2017^{[13]}$. Each institution should identify their level of risk for TB based on the geography and the time trends in the incidence of TB.

Clinical features such as fever of prolonged duration, typical chest radiograph findings, latent TB as diagnosed by a positive skin tuberculin test ${ }^{[14]}$ are highly suspicious for active TB infection. Akin to these clinical parameters a predictive model has been proposed for the diagnosis of TB in hospitalized patients ${ }^{[15]}$. Hence, the amalgam of high geographic prevalence along with time trends and symptomatic suspicion for active TB should be used towards the clinical acumen for early identification of high-risk cases.

An efficient testing protocol should be promptly paired with high clinical suspicion for TB to arrive at a definitive diagnosis. Pulmonary manifestations are the most common presentations. Typical chest radiologic findings have a major role in high clinical suspicion. However, it lacks specificity ${ }^{[16]}$. Chest X-ray and clinical features can be miss-interpreted as $\mathrm{CAP}^{[17]}$. Patients are frequently managed with the CAP targeted anti-microbial agents prior to diagnosis of $\mathrm{TB}^{[18]}$. Sputum microscopy with acid-fast staining, the initial step in diagnosis, is prompt and simple though at the expense of reliability due to low sensitivity ${ }^{[19]}$. The determination of pulmonary $\mathrm{TB}$, as per WHO recommendation, requires demonstration of bacteria in the sputum ${ }^{[20]}$. A sputum culture is reliable, preferred and gold standard for diagnosis of pulmonary TB. However, cultures come with the expense of time and take two to six weeks for them to be reported ${ }^{[19]}$. The nucleic acid amplification testing (NAAT) in sputum is a perfect blend of swiftness and reliability ${ }^{[21]}$ to derive a microbiological conclusion and to initiate the TB anti-microbial agents. The sensitivities differ in the sputum positive 
and negative TB patient ${ }^{[21]}$. There is an increase in the utilization of the NAAT over time ${ }^{[2]}$. There is no consensus on performing NAA for all hospitalized patients with suspected active TB. Centers for Disease Control recommends sputum NAAT on at least one sputum specimen in a patient with clinical signs suggestive of TB if the intended test changes the management by establishing the diagnosis ${ }^{[2,24]}$.

The clinical manifestations of TB for patients with HIV induced immunosuppression are unalike to immunocompetent host. HIV infected individuals are more likely to have extra-pulmonary TB as compared to HIV negative individuals ${ }^{[25]}$. Those with lower CD4 count are more likely to have extra-pulmonary $\mathrm{TB}^{[26]}$. A wide array of the differentials for the systemic illness in patients with advanced AIDS, as in our scenario, makes the diagnosis of the disseminated TB challenging. HIV infected individuals with TB can have atypical chest radiological findings ${ }^{[27]}$, with a small proportion of them with normal chest radiographs ${ }^{[24]}$. These adversities of atypical manifestations in conjunction with uncharacteristic radiological findings in patients with HIV hinders the early administration of anti-TB medication and hence can have worse outcomes in TBSS.

The culture of empiric treatment of TB differs across the globe. We assume that the incidence and prevalence of the disease guides the trend. The term "empiric" depicts two fundamental concepts. First is the presumptive treatment for a suspicious disease while awaiting the diagnosis. Second is the presumptive regimen while expecting the culture and sensitivity. Both parameters will affect the outcomes in sepsis. Molecular drug-resistance tests is prompt in detecting the drug resistance to isoniazid and rifampicin ${ }^{[28]}$ and can be used to titrate the anti-TB medication. However, given our limited experience and the rare entity of TBSS, it is difficult to opine on routine use of molecular drug-resistance tests for the management of TBSS. We performed a standard drug susceptibility testing in the management of our patients, and no drug resistance was identified.

Time is of the essence in the management of sepsis. The timing of the antibiotics is categorized as: (1) The timing from emergency room triage; and (2) Timing from the onset of sepsis or septic shock ${ }^{[29]}$. We carefully dissected Kethireddy et al ${ }^{[10]^{\prime}}$ s findings to review the "timing" of antibiotics administration and outcome of TBSS. The administration of the anti-tuberculous medications within $24 \mathrm{~h}$ of septic shock had improved mortality outcomes. In our cases, we used our clinical acumen to arrive at a prompt diagnosis of TB with appropriate susceptibility testing and timely administration of anti-TB medications. Despite optimal management, the outcomes were worst. These paradoxical findings ignited our curiosity to dig deeper into the pathogenesis of TBSS.

The systemic inflammatory response syndrome (SIRS) is defined by the host immune response in the form of four variables: Heart rate, respiratory rate, temperature and leukocytosis ${ }^{[30]}$. Given the variable immune response, there were some shortcomings to this definition of sepsis ${ }^{[31]}$. The inflammatory response can be severe enough to cause the circulatory and metabolic abnormality identified as a septic shock ${ }^{[32]}$. A prompt antibiotic administration targeting the inciting etiology of SIRS should lead to the prevention of septic shock. However, in our cases, we observed a paradoxical clinical worsening after administration of TB anti-microbial agents which may be explained by post-antibiotic immune reconstitution inflammatory syndrome (IRIS).

In our immunocompetent patients, the clinical course worsened after the initiation of anti-TB medications. In Kethireddy et al ${ }^{\left[100^{\prime}\right.}$ 's cases and other series ${ }^{[33]}$ of critically ill TB patients, we were unable to retrieve any similar observations of exacerbation of TB induced sepsis after antibiotics administration. In HIV coinfection the entity of IRIS is well recognized ${ }^{[34]}$. However, a paradoxical worsening during the anti-TB therapy may be linked to TB IRIS in the HIV negative population ${ }^{[35]}$. In HIV uninfected TB IRIS has an extra-pulmonary presentation and a chronic course ${ }^{[36]}$. The pathogenesis of TB IRIS in the non-HIV has been proposed ${ }^{[37]}$ but is not widely implemented in clinical practice. TB bacillary load has been identified as one of the risk factors for TB IRIS ${ }^{[3]}$. Prednisone has been utilized for the prevention of IRIS ${ }^{[39]}$. However, there is no clear consensus on steroid use in HIV negative patients, but can be considered ${ }^{[37]}$. A careful review of the clinical course in a few case reports and series depicts a similar clinical course $^{[40-42]}$. Hence, one should evaluate the possibility of IRIS in clinical scenarios of TBSS after TB antimicrobial administration.

Hyponatremia is another striking feature in our patients. Hyponatremia is prevalent in patients with pulmonary TB and severity correlates with extensive pulmonary parenchymal involvement and sputum positivity ${ }^{[43]}$. This correlation depicts the possibility of a high TB bacterial load in patients with hyponatremia. The pathogenies of hyponatremia, though poorly understood, has been linked to inappropriate secretion of the antidiuretic hormone ${ }^{[44]}$. Hyponatremia, though not statistically significant, has been related to high mortality rates in a patient with 
pulmonary $\mathrm{TB}^{[45]}$. But the rapid demise of the immunocompetent patients after $\mathrm{TB}$ antimicrobial administration begs to ask the question if there is any correlation of hyponatremia to high TB bacterial load and IRIS. To our knowledge, there is no study protocol exploring this specific rationale. Given the scarcity of data, we do want to conclude on this fact of hyponatremia and TB IRIS as an "observation" rather than "strong association".

The pathogenesis and clinical course of TB differ in immunocompromised patients with frequent atypical presentation and extrapulmonary dissemination ${ }^{[46]}$. TB may lead to worsening of HIV viremia and acceleration of immunosuppression ${ }^{[47]}$, hence increasing HIV related mortality ${ }^{[48]}$. In our patient with HIV co-infection, the onset of the septic shock was before the TB diagnosis and medication administration. Clinical findings were suggestive of the disseminated TB. The patient was on intravenous steroids prior to the anti-TB microbial administration. This reveals different pathophysiology then our immunocompetent hosts. The immune dysregulation is more likely from the bacterial infection rather than immune-reconstruction. However, advanced HIV could have contributed to the mortality despite anti-TB microbial administration.

\section{CONCLUSION}

TBSS is well recognized and widely reported though at risk of delayed diagnosis because of rare incidence in the United States. Antibiotics administration within 24 hours of the septic shock has been shown to improve mortality outcome in TBSS. High degree of suspicion and sputum NAA can be utilized towards rapid diagnosis and prompt administration of susceptible antibiotics. The pathogenesis of TBSS does differ in immunocompromised patients as opposed to immunocompetent. The paradoxical clinical worsening of patients with TB after susceptible antibiotics administration leading to TBSS does arise the possibility of TB IRIS. Our series though limited to 'observational remark' for TB IRIS as pathogenesis in an immunocompetent host does appeal a need for the future registry to evaluate this phenomenon.

\section{REFERENCES}

1 Rudd KE, Kissoon N, Limmathurotsakul D, Bory S, Mutahunga B, Seymour CW, Angus DC, West TE. The global burden of sepsis: Barriers and potential solutions. Crit Care 2018; 22: 232 [PMID: 30243300 DOI: $10.1186 / \mathrm{s} 13054-018-2157-z]$

2 Paoli CJ, Reynolds MA, Sinha M, Gitlin M, Crouser E. Epidemiology and Costs of Sepsis in the United States-An Analysis Based on Timing of Diagnosis and Severity Level. Crit Care Med 2018; 46: 18891897 [PMID: 30048332 DOI: 10.1097/CCM.0000000000003342]

3 Whiles BB, Deis AS, Simpson SQ. Increased Time to Initial Antimicrobial Administration Is Associated With Progression to Septic Shock in Severe Sepsis Patients. Crit Care Med 2017; 45: 623-629 [PMID: 28169944 DOI: 10.1097/CCM.0000000000002262]

4 Martin GS, Mannino DM, Eaton S, Moss M. The epidemiology of sepsis in the United States from 1979 through 2000. N Engl J Med 2003; 348: 1546-1554 [PMID: 12700374 DOI: 10.1056/NEJMoa022139]

5 Ferrer R, Martin-Loeches I, Phillips G, Osborn TM, Townsend S, Dellinger RP, Artigas A, Schorr C, Levy MM. Empiric antibiotic treatment reduces mortality in severe sepsis and septic shock from the first hour: Results from a guideline-based performance improvement program. Crit Care Med 2014; 42: 17491755 [PMID: 24717459 DOI: 10.1097/CCM.0000000000000330]

6 Buckman SA, Turnbull IR, Mazuski JE. Empiric Antibiotics for Sepsis. Surg Infect (Larchmt) 2018; 19 : 147-154 [PMID: 29341844 DOI: 10.1089/sur.2017.282]

7 Mayr FB, Yende S, Angus DC. Epidemiology of severe sepsis. Virulence 2014; 5: 4-11 [PMID: 24335434 DOI: $10.4161 /$ viru.27372]

8 Heemskerk D, Caws M, Marais B, Farrar J. Tuberculosis in Adults and Children 2015 [PMID: 26937536 DOI: $10.1007 / 978-3-319-19132-4]$

9 Sosa LE, Njie GJ, Lobato MN, Morris SB, Buchta W, Casey ML, Goswami ND, Gruden M, Hurst BJ, Khan AR, Kuhar DT, Lewinsohn DM, Mathew TA, Mazurek GH, Reves R, Paulos L, Thanassi W, Will L, Belknap R. Tuberculosis Screening, Testing, and Treatment of U.S. Health Care Personnel: Recommendations from the National Tuberculosis Controllers Association and CDC, 2019. MMWR Morb Mortal Wkly Rep 2019; 68: 439-443 [DOI: 10.1111/ajt.15512]

10 Kethireddy S, Light RB, Mirzanejad Y, Maki D, Arabi Y, Lapinsky S, Simon D, Kumar A, Parrillo JE, Kumar A; Cooperative Antimicrobial Therapy of Septic Shock (CATSS) Database Group. Mycobacterium tuberculosis septic shock. Chest 2013; 144: 474-482 [PMID: 23429859 DOI: 10.1378/chest.12-1286] Silva DR, Menegotto DM, Schulz LF, Gazzana MB, Dalcin PT. Mortality among patients with tuberculosis requiring intensive care: A retrospective cohort study. BMC Infect Dis 2010; 10: 54 [PMID: 20205952 DOI: 10.1186/1471-2334-10-54]

12 Talwar A, Tsang CA, Price SF, Pratt RH, Walker WL, Schmit KM, Langer AJ. Tuberculosis-United States, 2018. MMWR Morb Mortal Wkly Rep 2019; 68: 257-262 [DOI: 10.15585/mmwr.mm6811a2]

New York city bureau of tuberculosis control. Available from:

https://www1.nyc.gov/assets/doh/downloads/pdf/tb/tb2017.pdf

14 Frigati L, Maskew M, Workman L, Munro J, Andronikou S, Nicol MP, Zar HJ. Clinical Predictors of Culture-confirmed Pulmonary Tuberculosis in Children in a High Tuberculosis and HIV Prevalence Area. 
Pediatr Infect Dis J 2015; 34: e206-e210 [PMID: 26376315 DOI: 10.1097/INF.0000000000000792]

15 Wisnivesky JP, Kaplan J, Henschke C, McGinn TG, Crystal RG. Evaluation of clinical parameters to predict Mycobacterium tuberculosis in inpatients. Arch Intern Med 2000; 160: 2471-2476 [PMID: 10979058 DOI: 10.1001/archinte.160.16.2471]

16 Kumar N, Bhargava SK, Agrawal CS, George K, Karki P, Baral D. Chest radiographs and their reliability in the diagnosis of tuberculosis. JNMA J Nepal Med Assoc 2005; 44: 138-142 [PMID: 16751817]

17 Woodring JH, Vandiviere HM, Fried AM, Dillon ML, Williams TD, Melvin IG. Update: The radiographic features of pulmonary tuberculosis. AJR Am J Roentgenol 1986; 146: 497-506 [PMID: 3484866 DOI: 10.2214/ajr.146.3.497]

18 Wang M, Fitzgerald JM, Richardson K, Marra CA, Cook VJ, Hajek J, Elwood RK, Bowie WR, Marra F. Is the delay in diagnosis of pulmonary tuberculosis related to exposure to fluoroquinolones or any antibiotic? Int J Tuberc Lung Dis 2011; 15: 1062-1068 [PMID: 21740669 DOI: 10.5588/ijtld.10.0734]

19 WHO. World Health Organization. Early detection of tuberculosis: An overview of approaches, guidelines and tools. Geneva: World Health Organization 2011;

20 WHO. World Health Organization. Definitions and reporting framework for tuberculosis: 2013 revision (updated December 2014). Geneva: World Health Organization, 2013.

21 Greco S, Girardi E, Navarra A, Saltini C. Current evidence on diagnostic accuracy of commercially based nucleic acid amplification tests for the diagnosis of pulmonary tuberculosis. Thorax 2006; 61: 783-790 [PMID: 16738037 DOI: 10.1136/thx.2005.054908]

22 Tyrrell F, Stafford C, Yakrus M, Youngblood M, Hill A, Johnston S. Trends in Testing for Mycobacterium tuberculosis Complex From US Public Health Laboratories, 2009-2013. Public Health Rep 2017; 132: 56-64 [PMID: 28005481 DOI: 10.1177/0033354916679989]

23 Centers for Disease Control and Prevention. Available from: https://www.cdc.gov/tb/publications/guidelines/amplification_tests/reccomendations.htm

24 Ryu YJ. Diagnosis of pulmonary tuberculosis: Recent advances and diagnostic algorithms. Tuberc Respir Dis (Seoul) 2015; 78: 64-71 [PMID: 25861338 DOI: 10.4046/trd.2015.78.2.64]

25 Chaisson RE, Schecter GF, Theuer CP, Rutherford GW, Echenberg DF, Hopewell PC. Tuberculosis in patients with the acquired immunodeficiency syndrome. Clinical features, response to therapy, and survival. Am Rev Respir Dis 1987; 136: 570-574 [PMID: 3631730 DOI: 10.1164/ajrccm/136.3.570] Jones BE, Young SM, Antoniskis D, Davidson PT, Kramer F, Barnes PF. Relationship of the manifestations of tuberculosis to CD4 cell counts in patients with human immunodeficiency virus infection. Am Rev Respir Dis 1993; 148: 1292-1297 [PMID: 7902049 DOI: 10.1164/ajrccm/148.5.1292] Geng E, Kreiswirth B, Burzynski J, Schluger NW. Clinical and radiographic correlates of primary and reactivation tuberculosis: A molecular epidemiology study. JAMA 2005; 293: 2740-2745 [PMID: 15941803 DOI: 10.1001/jama.293.22.2740]

28 Chavalertsakul K, Boonsarngsuk V, Saengsri S, Santanirand P. TB-PCR and drug resistance pattern in BALF in smear-negative active pulmonary TB. Int J Tuberc Lung Dis 2017; 21: 1294-1299 [PMID: 28992819 DOI: $10.5588 /$ ijtld.17.0326]

29 Sterling SA, Miller WR, Pryor J, Puskarich MA, Jones AE. The Impact of Timing of Antibiotics on Outcomes in Severe Sepsis and Septic Shock: A Systematic Review and Meta-Analysis. Crit Care Med 2015; 43: 1907-1915 [PMID: 26121073 DOI: 10.1097/CCM.0000000000001142]

30 Marik PE, Taeb AM. SIRS, qSOFA and new sepsis definition. J Thorac Dis 2017; 9: $943-945$ [PMID: 28523143 DOI: $10.21037 /$ jtd.2017.03.125]

31 Vincent JL, Opal SM, Marshall JC, Tracey KJ. Sepsis definitions: Time for change. Lancet 2013; 381: 774-775 [PMID: 23472921 DOI: 10.1016/S0140-6736(12)61815-7]

32 Singer M, Deutschman CS, Seymour CW, Shankar-Hari M, Annane D, Bauer M, Bellomo R, Bernard GR, Chiche JD, Coopersmith CM, Hotchkiss RS, Levy MM, Marshall JC, Martin GS, Opal SM, Rubenfeld GD, van der Poll T, Vincent JL, Angus DC. The Third International Consensus Definitions for Sepsis and Septic Shock (Sepsis-3). JAMA 2016; 315: 801-810 [PMID: 26903338 DOI: 10.1001/jama.2016.0287]

33 Duro RP, Figueiredo Dias P, Ferreira AA, Xerinda SM, Lima Alves C, Sarmento AC, Dos Santos LC. Severe Tuberculosis Requiring Intensive Care: A Descriptive Analysis. Crit Care Res Pract 2017; 2017: 9535463 [PMID: 28250986 DOI: 10.1155/2017/9535463]

34 Meintjes G, Lawn SD, Scano F, Maartens G, French MA, Worodria W, Elliott JH, Murdoch D, Wilkinson RJ, Seyler C, John L, van der Loeff MS, Reiss P, Lynen L, Janoff EN, Gilks C, Colebunders R, International Network for the Study of HIV-associated IRIS. Tuberculosis-associated immune reconstitution inflammatory syndrome: Case definitions for use in resource-limited settings. Lancet Infect Dis 2008; 8: 516-523 [PMID: 18652998 DOI: 10.1016/S1473-3099(08)70184-1]

35 Cheng VC, Ho PL, Lee RA, Chan KS, Chan KK, Woo PC, Lau SK, Yuen KY. Clinical spectrum of paradoxical deterioration during antituberculosis therapy in non-HIV-infected patients. Eur J Clin Microbiol Infect Dis 2002; 21: 803-809 [PMID: 12461590 DOI: 10.1007/s10096-002-0821-2]

36 Lanzafame M, Vento S. Tuberculosis-immune reconstitution inflammatory syndrome. J Clin Tuberc Other Mycobact Dis 2016; 3: 6-9 [DOI: 10.1016/j.jctube.2016.03.002]

37 Sun HY, Singh N. Immune reconstitution inflammatory syndrome in non-HIV immunocompromised patients. Curr Opin Infect Dis 2009; 22: 394-402 [PMID: 19483618 DOI: 10.1097/QCO.0b013e32832d7aff]

38 Cheng SL, Wang HC, Yang PC. Paradoxical response during anti-tuberculosis treatment in HIV-negative patients with pulmonary tuberculosis. Int J Tuberc Lung Dis 2007; 11: 1290-1295 [PMID: 18034948 DOI: 10.1080/01902140701756778]

39 Meintjes G, Stek C, Blumenthal L, Thienemann F, Schutz C, Buyze J, Ravinetto R, van Loen H, Nair A, Jackson A, Colebunders R, Maartens G, Wilkinson RJ, Lynen L; PredART Trial Team. Prednisone for the Prevention of Paradoxical Tuberculosis-Associated IRIS. N Engl J Med 2018; 379: 1915-1925 [PMID: 30428290 DOI: 10.1056/NEJMoa1800762]

40 Bridges DA, Bedimo RG. Severe tuberculosis sepsis in an immunocompetent patient. Am J Med 2006; 119: el1-e14 [PMID: 16490454 DOI: 10.1016/j.amjmed.2005.08.033]

41 Nidadavolu VG, Shah M, Kuriti M. Tuberculosis Presenting As "SEPSIS"! -An Interesting Presentation Of Miliary Tuberculosis. ATS J 2013; A3213-A3213

42 Michel P, Barbier C, Loubière Y, Hayon JH, Ricôme JL. Three cases of septic shock due to tuberculosis without HIV pathology. Intensive Care Med 2002; 28: 1827-1828 [PMID: 12447531 DOI: 10.1007/s00134-002-1526-9]

43 Bokam BR, Badikillaya VU. Prevalence of hyponatremia in pulmonary tuberculosis-A pilot study from a 
tertiary care center in south India. Int J Med Sci Public Health 2017; 6: $75-79$ [DOI:

10.5455/ijmsph.2017.20062016560]

44 Lee $\mathbf{P}$, Ho KK. Hyponatremia in pulmonary TB: Evidence of ectopic antidiuretic hormone production. Chest 2010; 137: 207-208 [PMID: 20051406 DOI: 10.1378/chest.09-0405]

45 Vargasa T, Morales-Garza LA, Maya R, Llamas-Lopez A. Available from: https:/idsa.confex.com/idsa/2018/webprogram/Paper72122.html

46 Aaron L, Saadoun D, Calatroni I, Launay O, Mémain N, Vincent V, Marchal G, Dupont B, Bouchaud O, Valeyre D, Lortholary O. Tuberculosis in HIV-infected patients: A comprehensive review. Clin Microbiol Infect 2004; 10: 388-398 [PMID: 15113314 DOI: 10.1111/j.1469-0691.2004.00758.x]

47 Garrait V, Cadranel J, Esvant H, Herry I, Morinet P, Mayaud C, Israël-Biet D. Tuberculosis generates a microenvironment enhancing the productive infection of local lymphocytes by HIV. J Immunol 1997; 159: 2824-2830 [PMID: 9300705]

48 Whalen C, Horsburgh CR, Hom D, Lahart C, Simberkoff M, Ellner J. Accelerated course of human immunodeficiency virus infection after tuberculosis. Am J Respir Crit Care Med 1995; 151: 129-135 [PMID: 7812542 DOI: 10.1164/ajrccm.151.1.7812542] 


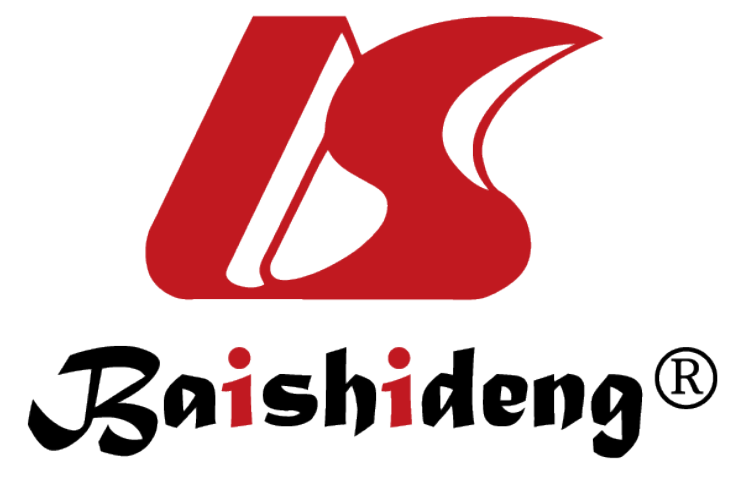

Published By Baishideng Publishing Group Inc

7041 Koll Center Parkway, Suite 160, Pleasanton, CA 94566, USA

Telephone: +1-925-2238242

Fax: +1-925-2238243

E-mail: bpgoffice@wjgnet.com

Help Desk: https://www.f6publishing.com/helpdesk

https://www.wjgnet.com

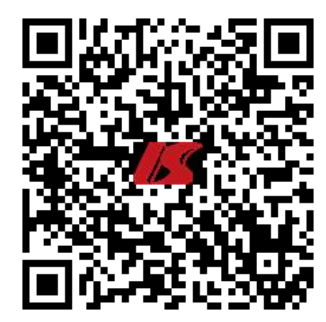

